\title{
eLabFTW as an Open Science tool to improve the quality and translation of preclinical research [version 1; peer review: 1
}

\section{approved with reservations]}

\section{Michael Hewera (Di)1, Daniel Hänggi1 , Björn Gerlach (Di) 2*, Ulf Dietrich Kahlert(D)1,3*}

${ }^{1}$ Clinic for Neurosurgery, Medical Faculty, Heinrich Heine University Dusseldorf, Düsseldorf, NRW, 40225, Germany

2PAASP GmbH, Heidelberg, BW, 69118, Germany

${ }^{3}$ Beijing Neurosurgical Institute, Beijing, China

* Equal contributors

V1 First published: $16 \mathrm{Apr} 2021,10: 292$

https://doi.org/10.12688/f1000research.52157.1

Second version: 27 May 2021, 10:292

https://doi.org/10.12688/f1000research.52157.2

Latest published: 02 Aug 2021, 10:292

https://doi.org/10.12688/f1000research.52157.3

\section{Abstract}

Reports of non-replicable research demand new methods of research data management. Electronic laboratory notebooks (ELNs) are suggested as tools to improve the documentation of research data and make them universally accessible. In a self-guided approach, we introduced the open-source ELN eLabFTW into our lab group and, after using it for a while, think it is a useful tool to overcome hurdles in ELN introduction by providing a combination of properties making it suitable for small preclinical labs, like ours. We set up our instance of eLabFTW, without any further programming needed. Our efforts to embrace open data approach by introducing an ELN fits well with other institutional organized ELN initiatives in academic research.

\section{Keywords}

Quality Management, Electronic Lab Notebook, ELN, Open Science, Reproducibility, Transparency

This article is included in the Research on

Research, Policy \& Culture gateway.

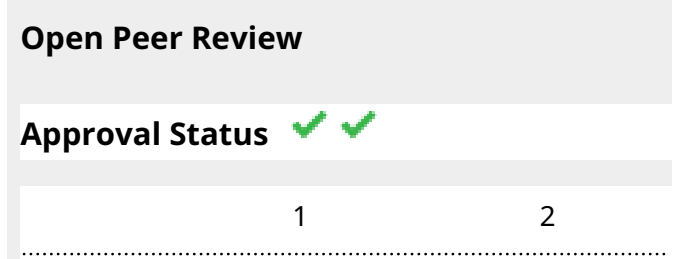

version 3

(revision)

02 Aug 2021

version 2

(revision)

27 May 2021

version 1

16 Apr 2021

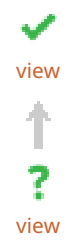

1. Sam Parsons ID, University of Oxford,

Oxford, UK

2. Amiram Goldblum (D), University of Oxford,

Oxford, UK

University of Oxford, Oxford, UK

Any reports and responses or comments on the article can be found at the end of the article. 
Corresponding authors: Michael Hewera (michael.hewera@med.uni-duesseldorf.de), Ulf Dietrich Kahlert (Ulf.Kahlert@med.uniduesseldorf.de)

Author roles: Hewera M: Conceptualization, Methodology, Project Administration, Resources, Visualization, Writing - Original Draft Preparation, Writing - Review \& Editing; Hänggi D: Methodology, Resources, Writing - Review \& Editing; Gerlach B: Conceptualization, Methodology, Writing - Review \& Editing; Kahlert UD: Conceptualization, Methodology, Project Administration, Resources, Writing Original Draft Preparation

Competing interests: BG is an employee and shareholder at PAASP GmbH. All other authors declare that they have no competing interests.

Grant information: The author(s) declared that no grants were involved in supporting this work.

Copyright: $\odot 2021$ Hewera $\mathrm{M}$ et al. This is an open access article distributed under the terms of the Creative Commons Attribution License, which permits unrestricted use, distribution, and reproduction in any medium, provided the original work is properly cited.

How to cite this article: Hewera M, Hänggi D, Gerlach B and Kahlert UD. eLabFTW as an Open Science tool to improve the quality and translation of preclinical research [version 1; peer review: 1 approved with reservations] F1000Research 2021, 10:292

https://doi.org/10.12688/f1000research.52157.1

First published: 16 Apr 2021, 10:292 https://doi.org/10.12688/f1000research.52157.1 


\section{Introduction}

In the light of reports of non-replicable research results, new ways to strengthen an open science approach for better transparency, reproducibility and traceability are needed. ${ }^{1}$ Reproducibility meaning, that following the proper formatted documentation, any other lab group with the necessary equipment and personnel should be able to obtain the same results of any experiment performed. ${ }^{2}$ An easy way to increase the legibility, tidiness, and overall quality of documentation, can be the introduction of an electronic laboratory notebook (ELN) in a research unit. Publishing the ELN entries in an unaltered toegether with the manuscript enhances open science by creating transparency and will ultimately support reproducibilty. ${ }^{3}$ Here, ELNs will help in the context of research data management to make the entire life cycle of data transparent and better integrate it in the documentation process. ${ }^{4}$ The pros and cons of using ELNs have been discussed for a long time. The main fields of discussion are usability, accessibility, costs, time effort and data safety. ${ }^{5,6}$ In our experience, the use of an ELN has many benefits towards traditional paper-based notebooks, but it is often a challenge to convince researchers of the advantages of using an ELN. We believe that the use of eLabFTW, and open source software, is a particularly good approach to overcome this hurdle and ensure high research data quality in small, preclinical research groups like ours.

eLabFTW is a lightweight general-purpose ELN. It consists of an Experiment and a Database section, whereas the Experiment section is designed to create and edit experimental documentation and link it to entries of the Database section. These can be set by the administrator to be any type of object (Chemicals, cell lines, equipment, etc.). These two sections are complemented by a Teams section, in which equipment can be booked in a scheduler and experiment templates can be shared. Finally, eLabFTW provides an extensive search tool. ${ }^{7}$

\section{Advantages of ELN on experimental and lab management level}

Although there are some disadvantages in using ELNs, ${ }^{6,8}$ we believe that the advantages outweigh those by far. Especially in aspects regarding open science and reproducibility, data safety and availability, as well as transparency and as a tool for thorough standardization. Additionally, to those most important factors, there are other advantages in using ELNs: digital recording of research data makes it much easier for researchers to find their older data as well as data of other users. Also, the sharing of data is simplified, which makes discussing, cooperation and troubleshooting of experimental procedures much easier. ELNs contain time saving mechanisms, such as automatic import of data, the possibility of linking experiments and resources, and the usage of templates for standard experiments, which can additionally simplify the planning of experiments. Furthermore, most ELNs can also contribute to the simplification of lab management aspects as inventory management, sample tracking, communication, and digitalization of paperwork. Finally, in our experience data recording and documentation is done more carefully, when researchers have in mind, that their records are visible to others.

\section{Hurdles of introducing ELN}

Nowadays, most data in life science are digital data. Thorough recording, documentation and annotation of this data is difficult in a traditional paper laboratory notebook. Using ELNs will not only ensure the data to be recorded in an efficient and comprehensive way but will also facilitate findability and reusability. Additionally, the possibility to create templates, standard operating procedures (SOPs) and predefined workflows in ELNs is time saving in routine lab work and sharing of knowledge. In combination with the search function, an ELN can become a pool of knowledge for the lab group and even for people outside of it. ${ }^{4}$

However, as discussed in other sources, the overall adoption of ELNs is rather low in the academic field, as there are many perceived disadvantages in the use of ELN, as well as hurdles that must be overcome. These hurdles include the costs of obtaining the software and maintaining the necessary infrastructure, the time needed to implement the ELN and adapt the lab habits, as well as the fear of data being stored in a cloud on a foreign server. ${ }^{6}$ Another point that is important when working with primary samples of patient data is the protection of personal data (e.g., patient information) from unauthorized access. ${ }^{6}$ Other scientists fear that the software will be discontinued in the future, making it necessary to search for a new ELN, with the uncertainty whether the data from the previous system can still be accessed and imported to the new system. ${ }^{6}$

\section{eLabFTW has a combination of features making it a powerful tool to overcome hurdles}

The open-source ELN eLabFTW (https://www.elabftw.net/) was developed to provide lab groups with a free to use ELN. The developers of eLabFTW being researchers themselves, provided a general purpose ELN with limited features, but a powerful database and search function. Making it easy to learn and use, yet very suitable for all kinds of research fields. It consists of an Experiment section, used for documenting experiments with a simple built in text editor and a Database section, which can be used to create entries for all kinds of items like plasmids, enzymes, primers, etc. by using the same text editor. Furthermore, these items can be imported automatically via comma separated value (CSV) files by the admin. 
Subsequently, these items can be directly referenced from the experiment section. Experiments themselves also can be linked to other experiments, making it easy to keep a seamless documentation of projects and experiments. eLabFTW is built according to the open data principle, providing open-source code, as well as providing on board functions to easily share experiment and database entries with people in- and outside of eLabFTW via direct links, or export to PDF or ZIP files.

In our opinion, eLabFTW is well suited to overcome the aforementioned hurdles of ELN implementation in small preclinical labs, as well as in other environments and lead to an improvement of research data transparency and findability. Being an open-source software, there are no license or other costs to pay when acquiring eLabFTW. In addition, even if the development and support of the software is discontinued, the source code will always be available, preventing users to search for a new software solution. eLabFTW does not use any proprietary parts of code, so this property applies to the whole code of eLabFTW. Some other ELNs are open source but depend on pieces of proprietary third-party code. In addition, when needed, the code of eLabFTW can be adapted to individual needs, by trained IT-Staff in the organization. Providing the option to use Docker Containers, a kind of virtual machine, which contains all the necessary software to run eLabFTW. eLabFTW can be installed even by a person with only intermediate IT knowledge, when following the official documentation. ${ }^{9}$ The initial setup takes less than 30 minutes, on a Windows computer, as tested by us. The setup works on a server or even a simple computer running Linux, Windows or MacOS. That ability for local installation makes eLabFTW suitable for critical data and enables data storages independently from the availability of cloud services offered by other ELN companies. This ensures high data safety, as it prevents access to data from outsiders and protects from using services with uncompliant privacy policies. Backup of data can be set up using a simple script ${ }^{9}$ and the backup files can be stored on a second hard drive, or on other machines, like a Network Associated Storage. eLabFTW offers a full text search engine, meaning it searches for all elements of the database, as well as in the written text of entries, making it easy to find older research data from yourself, and others. Additionally, eLabFTW contains a powerful search tool, which allow users to search for entries with specific properties. All entries in the experiment section of eLabFTW can be timestamped after RFC 3161 standard, allowing for full audit trail documentation. In addition, all revisions in any entry are tracked, so that it can be proved that no data were altered. Every item in eLabFTW is identified by a unique identifier (eLabID), which is independent from the given title. Hence, all objects can be unambiguously identified. Finally, all entries in eLabFTW can be shared via a unique link with people inside or outside the lab group, if eLabFTW is running on a computer with internet connection.

The features mentioned above are the most outstanding differences between eLabFTW and a traditional paper-based lab notebook, or other ELNs. However, eLabFTW provides some more useful features, that can be beneficial for a lab. Firstly, it offers a simple interface with just a text editor and some additional features like file upload and the integration of image files into the text. Furthermore, items in eLabFTW are marked by tags, instead of being ordered in a folder structure. In our experience this works much better, because entries can be sorted with multiple tags, in contrast to just be in one single folder. Any item in the database can be exported to different formats (pdf, zip, json) to be shared or stored externally. The visibility for each item can be restricted to oneself, a specific group of people or set to public. This might be an important feature to secure rights of research. Lastly, eLabFTW provides an API that can be accessed with a specifically created python library. For example, this is useful for automatically upload raw data to eLabFTW.

\section{Implementation of eLabFTW in our lab}

The implementation of an ELN was supposed as the first step of a bigger project of increasing the research data quality in our lab, as described in a prior publication. ${ }^{10}$ For this, we tested different trials of commercially and freely available ELNs. Our focus was on the usability rather than on the features, as we wanted to make transition to electronic documentation as easy as possible and many features offered by more expensive ELNs are not needed by our group. After initial testing, two commercial ELNs and eLabFTW came into closer selection. We got the notice that two ELNs in this selection are already hosted by our university, this being Labfolder and eLabFTW. After a two-week period during which both lab books were tested in a small group, we decided to introduce eLabFTW to the whole lab. Although Labfolder has some features that eLabFTW does not have (support of including Excel and Word files, adaptable page layout), we decided to implement eLabFTW because of the much quicker processing times, open-source format and in our opinion a much easier usability. After introducing eLabFTW to every lab member, we decided about the most useful setup for our lab environment, to make it suitable for our QM-Project.

Specific setup of eLabFTW in our lab (Figure 1)

Before the setup of eLabFTW, we already started to introduce SOPs for our basic lab procedures. Using the database of eLabFTW proved to be a good way to distribute new versions of SOPs to every lab member effectively. As a first step, all existing SOPs were transferred into eLabFTW. The first new SOP described how to use eLabFTW. This SOP describes the form of documenting and time stamping of experiments, as well as linking database items. Secondly, we imported our 


\section{eLabFTW}

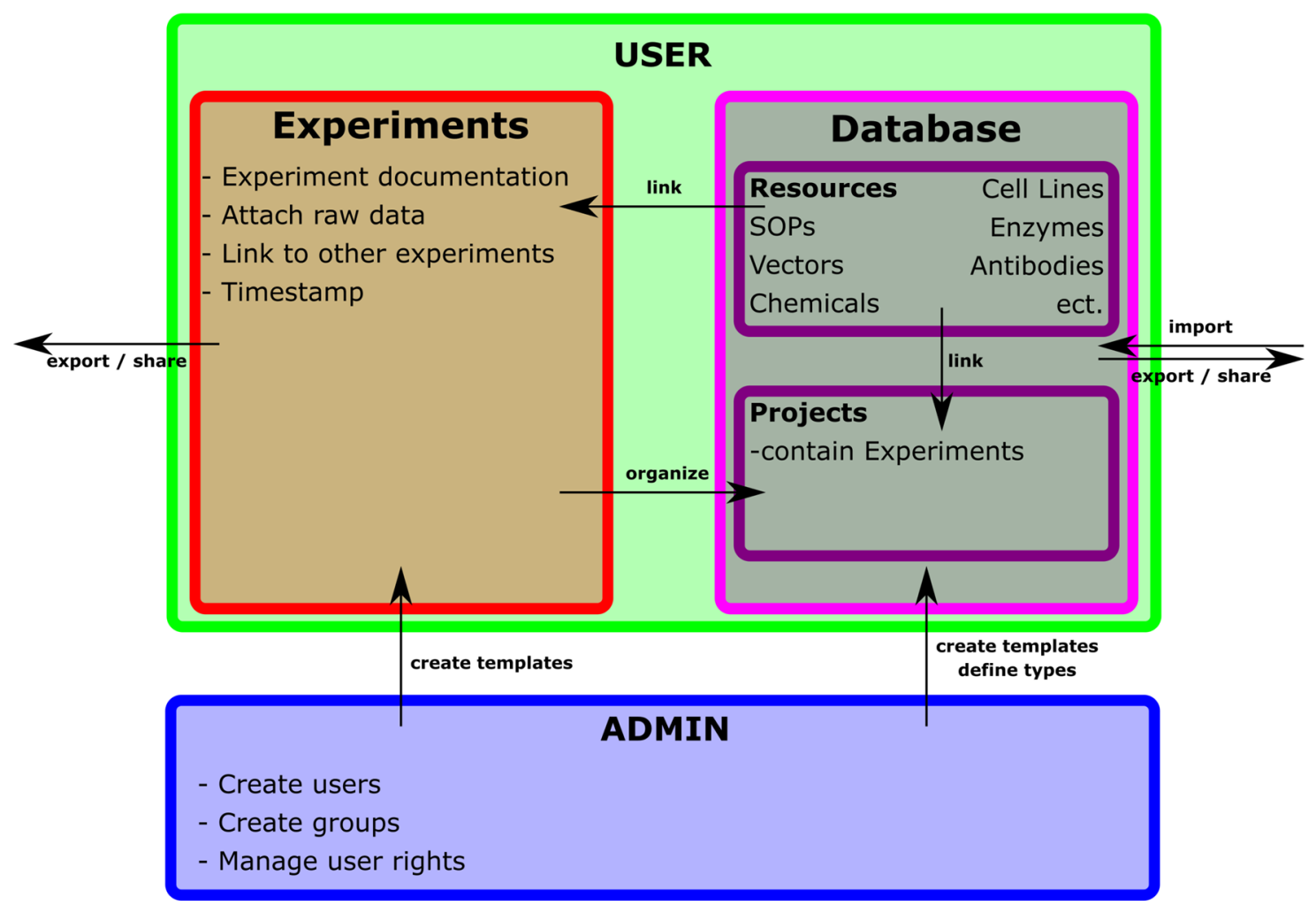

Figure 1. Configuration of eLabFTW as being used by our workgroup.

complete database of vectors, primers, antibodies, enzymes, chemicals, consumables, and equipment, as well as cell lines into eLabFTW, together with the matching documentation as there are vector maps, material safety datasheets, primer sequences and melting temperature, antibody datasheets, restriction sites of restriction enzymes, cell line properties, handbooks of machinery, and so on. We also use the database to create project items, that all experiments belonging to that project can be linked to, to establish a timeline of experiments performed in that project. Lastly, we use eLabFTW to upload meeting minutes and documentation of other events.

The visibility in our instance of eLabFTW can be set to the whole team, or subgroups working on specific projects, that we create as needed. In our lab we do not use the booking function of lab equipment, after we found it too inconvenient after a short test period.

As described in one of our previous publications, ${ }^{10}$ the ELN was also supposed to be used for one-on-one project communication in form of interactive weekly progress reports. This approach was ineffective, as it consumed too much time, lead to misunderstandings and was unsuitable for the discussion of some specific problems. Consequently, we switched back to personal, verbal meetings for project planning. In our experience, ELN-reporting is only suitable for one directional communication. Still this possibility of one-directional way of communication showed an unexpected advantage during the still ongoing COVID-19 pandemic. Due to electronic recording of research results and protocols, less face-to-face communication was necessary, which helped to keep the infection risk on a low level.

Our effort embeds well in current initiatives from leading biomedical institutions, as the RE-PLACE Project at the Berlin Institute of Health (BIH), ${ }^{11}$ which is an institution-wide programme for adopting ELN software at Charite and BIH, by providing free licenses to the ELN Labfolder, as well as introductory courses and support. This whole project is combined with an evaluation study to see if ELN usage really is beneficial for fulfilling needs of researchers and to improve the quality and transparency of research documentation and data management.

Another project, that our lab is involved in, is the EQIPD quality system (QS). ${ }^{12}$ Our efforts on eLabFTW fit very well with the requirements of this first quality system for preclinical research. Implementing and maintaining an ELN supports 
the implementation and maintenance of several Core Requirements set forth by the EQIPD QS, i.e. those on data documentation and transparency. In fact, our efforts to make our science open and sharable are a major contribution towards certification of our internal processes. Hence, an ELN should be considered as one of the pillars towards quality processes in a small research lab, especially when it integrates seamless in the daily lab routine as eLabFTW does.

There are also the more general ELN guides from the Harvard Medical School Information Technology department ${ }^{13}$ and the BIH. ${ }^{14}$

In a forthcoming visionary standpoint, we think that stringent ELN implementation in more working units is facilitating the reduction of hurdles for interaction across disciplines, such as the interaction of basic scientists and patient-treating clinical faculty. We hypothesize this due to the fact that data can be rapidly and remotely available at any given time, not restricted to often incompatible time schedule of the participating stakeholders. Moreover, a growing body of large datasets will be summarized and presented in a more comprehensive and convenient way as compared to local storage solutions on core faculty servers or hidden folders in a given server infrastructure. Thereby, further exchanging interaction to data scientists and wet lab scientists is possible. Laboratory automation, as a standard for many work processes in industry and routine diagnostic labs, is embracing its way in many academic labs ${ }^{15}$ such as in our lab occurred for a liquid handling robotics. ${ }^{16}$ Direct linking of ELN to machine output, without human operator data handling step in between, saving the data file in a time stamped and logged version will minimize error introduction in parallel to increase transparency of the project. Moreover, linking lab automation to ELN with a time scheduling algorithm may improve economic effectivity of the working unit. ${ }^{17}$ Those aspects are only a few cornerstones that shall be a focus of operations developments of a state-of-the-art translational research lab, currently underway in the lab of neurosurgery at the University Clinic Düsseldorf.

\section{Data availability}

No data is associated with this article.

1. Baker M: Is there a reproducibility crisis? Nature. 2016; 533(7604): 452-454.

2. Diggle PJ, Zeger SL: Editorial. Biostatistics. 2010; 11:3, p375. Publisher Full Text

3. Nielsen M: An informal definition of open science. The OpenScience Project. 2011.

Reference Source visited on 22.02.2021

4. Adam B, Lindstädt B: ELN-Wegweiser. 2nd edition 2020. Publisher Full Text

5. Kihlén M: Electronic lab notebooks - do they work in reality? DDT. 2005; 10: 18.

PubMed Abstract | Publisher Full Text

6. Gerlach B, Untucht Ch, Stefan A: Electronic Lab Notebooks and Experimental Design Assistants. Handb Exp Pharmacol. 2020; 257: 257-275.

PubMed Abstract | Publisher Full Text

7. Carpin MA, Piel M: eLabFTW: An open source laboratory notebook for research labs. JOSS 2017; 2(12): 146. Publisher Full Text

8. Walsh E, Cho I: Using Evernote as an Electronic Lab Notebook in a Translational Science Laboratory. J Lab Autom. 2012; 18(3): 229-234.

PubMed Abstract | Publisher Full Text

Reference Source visited on 22.02.2021

10. Hewera $M$, et al.: An inexpensive and easy-to-implement approach to a Quality Management System for an academic research lab. F1000Res. 2020; 30(9).

PubMed Abstract | Publisher Full Text | Free Full Text

11. Wetzel C, Pohlenz P, Schirmer D: Wissenschaft zwischen Konkurrenz und Kooperation - Zum Potenzial kooperationsfördernder Managementinstrumente. DUZ OPEN. Publisher Full Text

12. Bespalov A, et al.: Introduction to the EQIPD Quality System. OSF 2007. Publisher Full Text

13. Loveluck J: Finding the Right Electronic Lab Notebook with the Corey Lab. Reference Source visited on 22.02.2021

14. Dirnagl U, Przesdzing I: A pocket guide to electronic laboratory notebooks in the academic life sciences. F1000Res. 2016; 5: 2. PubMed Abstract | Publisher Full Text | Free Full Text

15. Lippi G, Da RG: Advantages and limitations of total laboratory automation: a personal overview. CCLM 2019; 75(6). PubMed Abstract | Publisher Full Text

16. Vargas-Toscano A, et al.: Robot technology identifies a Parkinsonian therapeutics repurpose to target stem cells of glioblastoma. CNS Onkol. 2020; 9 (2). PubMed Abstract | Publisher Full Text | Free Full Text

17. Chung HJ, Song YK, Hwang SH, et al.: Experimental fusion of different versions of the total laboratory automation system and improvement of laboratory turnaround time. JCLA 2018; 32(5). PubMed Abstract | Publisher Full Text | Free Full Text 


\section{Open Peer Review}

\section{Current Peer Review Status: ?}

\section{Version 1}

Reviewer Report 06 May 2021

https://doi.org/10.5256/f1000research.55395.r84047

(c) 2021 Parsons S. This is an open access peer review report distributed under the terms of the Creative Commons Attribution License, which permits unrestricted use, distribution, and reproduction in any medium, provided the original work is properly cited.

\section{Sam Parsons}

Department of Experimental Psychology, University of Oxford, Oxford, UK

The manuscript describes the open-source electronic lab notebook eLabFTW and the authors experience with implementing this into their workflow. I see this as a potentially useful contribution to the literature, in part because I am broadly positive about the use of ELNs. The majority of my comments are based around improving the clarity of the manuscript - I have numbered suggestions for ease.

1. The main point (which ties in to many of the below suggestions for clarity) I am not clear on after reading the manuscript, is the framing or the take-home message I should be left with. The content included is informative, but moves between; support or advocacy for the eLabFTW program, reflections on the groups experiences implementing it, and reflections on the good work for open science the authors groups and institutions are doing.

Throughout, it feels that these aims are merged together, which makes the flow of the paper difficult to follow. I have several general suggestions that could help improve this clarity:

1. Consider more subheadings to distinguish the message of each subsection. At a minimum, I think it would be valuable to separate or clearly distinguish sections. e.g. descriptions of eLabFTW's technical details and instructions from the authors reflections on implementing eLabFTW. Greater use of subheadings would help the flow of the manuscript and reader clarity.

2. Given that the abstract describes the paper as providing a self-guided approach to using eLabFTW, this section could benefit from more detail. Reading the abstract, I thought (though this may be just my reading) that this would be the core element of the paper but this section was quickly moved into more general comments on other initiatives. Further detail, and examples such as the groups own ELN, would make this a much more useful contribution.

3. Consider removing some references to work specific to the authors groups/institutions - or, revise so that it can be made relevant to the readers context in using ELNs. For example, as a reader I did not find the closing sentence to be useful to my understanding of the eLabFTW tool "Those aspects are only a few cornerstones that shall be a focus of operations developments of a state-of-the-art 
translational research lab, currently underway in the lab of neurosurgery at the University Clinic Düsseldorf". The sentences describing the good work towards openness will be maximally useful to the reader if they are situated within how they can implement these tools themselves, otherwise they are not necessary to the manuscript and distract from the main content.

2. The manuscript mentions 'As described in one of our previous publications' several times. My original suggestion was that more description of what was discussed would be valuable in this manuscript, else the reader is missing potentially vital information to this context. Having now read the cited paper, I am less clear of the purpose of the "Implementation of eLabFTW in our lab" section of this manuscript, which in turn leaves me less clear of the purposes of this manuscript, which related to my first point also. Perhaps the authors can more clearly differentiate between the contribution both papers make and what this manuscript adds in particular?

The following comments relate to more specific sections of the manuscript.

1. In the opening paragraph, it may be useful to differentiate between the authors definition of reproducibility and replicability. Later in the paragraph focus is placed mainly on data management, so I thought that computational reproducibility was the focus (same data + same analyses = same results) whereas the definition used was what others might describe as replicability (new data with the same procedures = same results). Not that I have a problem with the definition used, but the clarity for the focus or benefits of ELNs could be improved with this.

2. Minor typo: "unaltered toegether" I think that a word might also be missing here, perhaps 'in an unaltered format together with...'?

3. "The pros and cons of using ELNs have been discussed for a long time" - Perhaps tie this in to the following two sections, or provide citations here?

4. I suggest moving around some of the text in the advantages and hurdles sections - it was slightly confusing to read as the advantages section first highlighted disadvantages and the hurdles also starts with an entire paragraph on the benefits. As mentioned above, citing examples that highlight the benefits would be valuable here too.

5. Perhaps the authors could be clearer about the focus on life sciences. Given that this is the context the ELN was implemented in, it makes sense. But, it might be useful to highlight or discuss further the use of ELNs in other contexts, or state that this paper focuses on the life sciences solely.

6. I suggest splitting the long paragraph discussing the open-source aspect of eLabFTW at least into a paragraph on open-source and a paragraph on the set up (further use of subheadings would assist here too).

\section{I suggest removing the SOP acronym as it is not needed.}

8. I was not sure of the purpose of the paragraph on EQIPD, it did not feel relevant to the ELN discussion.

9. Perhaps the paragraph on other guides for ELNs could be integrated to somewhere else in 
the manuscript?

In the interest of openness I always sign my reviews,

Sam Parsons

Is the topic of the opinion article discussed accurately in the context of the current literature?

Yes

Are all factual statements correct and adequately supported by citations? Partly

Are arguments sufficiently supported by evidence from the published literature? Yes

Are the conclusions drawn balanced and justified on the basis of the presented arguments? Yes

Competing Interests: No competing interests were disclosed.

Reviewer Expertise: Openness and reproducibility, cognitive affective, psychometrics.

I confirm that I have read this submission and believe that I have an appropriate level of expertise to confirm that it is of an acceptable scientific standard, however I have significant reservations, as outlined above.

Author Response 12 May 2021

Michael Hewera

Dear Dr. Parsons,

I (in the name of my co-authors, as well) thank you for reviewing our publication and for your valuable feedback.

We tried to implemented most of your suggestions in the new version of our publication.

These are (not only)

- Condensing the part about pros and cons of ELNs and sortig it

- Specifying references to "prior publication"

- moving text, and creating new headings/sub-headings

- Better explain the context of eLabFTW and EQIPD

- Streamline the last chapter

Best regards,

Michael Hewera

Competing Interests: No competing interests were disclosed. 
The benefits of publishing with F1000Research:

- Your article is published within days, with no editorial bias

- You can publish traditional articles, null/negative results, case reports, data notes and more

- The peer review process is transparent and collaborative

- Your article is indexed in PubMed after passing peer review

- Dedicated customer support at every stage

For pre-submission enquiries, contact research@f1000.com 